\title{
Hydration forces at solid and fluid biointerfaces
}

\author{
Buddha R. Shrestha, Xavier Banquy \\ Canada Research Chair in Bio-Inspired Materials and Interfaces, Faculty of Pharmacy, Université de \\ Montréal, PO Box 6128, Montréal, QC H3C 3J7, Canada \\ * Corresponding author: xavier.banquy@umontreal.ca
}

\begin{abstract}
We review the different molecular mechanisms giving rise to the repulsive hydration force between biologically relevant surfaces such as lipid bilayers and bio-ceramics. As we will show, the hydration force manifests itself in very different and subtle ways depending on the substrates. Soft, mobile surfaces such as lipid bilayers tend to exhibit monotonic, decaying hydration force, originated from the entropic constriction of the lipid head groups. Solid surfaces on the other hand, tend to exhibit a periodic oscillatory hydration force, originated from the surface induced polarization of water molecules. In this review we will describe both subtle faces of this important interaction by first describing the early experiments performed on solid surfaces and their interpretation by recent simulation studies. Then we will describe the hydration force between fluid interfaces such as bilayers and explain how experimentally researchers have unraveled the dominant role of the lipid head groups conformation.
\end{abstract}

\section{INTRODUCTION}

For decades, it was perceived that the Derjaguin Landau Verwey and Overbeek (DLVO) theory[1,2], a combination of repulsive electrical double layer forces and attractive van der Waals forces, is able to describe the properties of wide range of colloids and bio-colloids. According to this theory, at separations below 2-4 nm, attractive van der Waals forces always dominates over the repulsive double layer forces leading to an adhesive contact. However, several phenomena (re-peptization, hydrophobic colloids, and silica particles) were found not to obey this theory as these materials neither coagulated nor swelled as predicted by DLVO theory. Thus, the idea of a repulsive force, the hydration force, acting at a few nanometer separation distance, overcoming the van der Waals primary adhesive minimum arose. These observations, yet, were not sufficient to prove the existence of an additional force simply because other repulsive contributions such as the Stern layers compression or the possible presence of steric repulsive interactions had to be ruled out [3]. Frens and Overbeek [4, 5] proposed a model which explained the removal of the primary adhesive minimum by shifting the hard wall cut off to a finite separation of $D=D_{0}+2 d$, where $D_{0}$ was the zero distance and $d$ the diameter of ions. This theory showed that the net repulsion could continue to rise steeply as the inter-particle distance separation decreased to contact even when the surface charge density or potential was low. After this first intent, the necessity of directly measuring the interaction forces vs. separation distance was the subject of intense research in order to experimentally verify if the force laws measured can be fitted using DLVO-Stern model or if it requires some extra force to be fitted. In what 
follows, we will describe the experimental characterization of the hydration forces, in two main systems, namely lipid membranes and solid substrates, the apparent discrepancies in the experimental observations that have emerged and the common ground between different researches. We describe the various experimental methods that are still used to determine such forces and continue with a detailed description of the results obtained from Surface Force Apparatus (SFA).

\section{FORCE MONITORING TECHNIQUES}

Several direct force measuring techniques have been developed till date. Some were devised for specific purposes while others have been implemented for a broad range of applications. The first technique to provide the quantitative data into the short range hydration forces is the Osmotic Pressure (OP) technique. OP is able to measure the repulsive forces in aqueous solutions between surfactants or lipid bilayers [6-8], aligned clay sheets [9, 10] and aligned macromolecules [11]. In this method, osmotic pressure is applied to the aqueous sample and the equilibrium spacing between surfaces is monitored by X-rays. This technique, however, is unable to measure interaction forces at longer range and is thus limited to only the repulsive parts of the force laws. OP is not able to detect any adhesive forces. Later, SFA was developed by Tabor, Winterton and Israelachvili [12-14] to measure the van der Waals forces between molecularly smooth mica surfaces in air or vacuum. Israelachvili further modified the apparatus and enabled it to monitor forces in liquid medium as well. This opened the gateway to the broad field of modern surface science for instance rheology [15], tribology [16], sensing [17], electrochemistry [18], corrosion [19].

Figure 1A shows the schematic representation of the SFA apparatus. A white light beam is directed through two curved discs covered with back-silvered mica sheets of thickness 2 to 5 $\mu \mathrm{m}$. The silver thickness on mica is about $45 \mathrm{~nm}$ to $50 \mathrm{~nm}$ to allow at least $95 \%$ reflection. The discs are placed in cross cylinder geometry creating a three layer interferometer. The interference fringes emerging from the apparatus are called fringes of equal chromatic order (FECO). The transmitted light is directed to a spectrometer for further analysis of FECO fringes. The interaction force, $F$, acting between the surfaces is measured by the deflection of a spring on which one of the surfaces is mounted [20]. This technique has distance and force resolutions of 1 $\AA$ and $1 \mu \mathrm{N}$ respectively [21]. The detailed description of the instrument is given in ref. [22]. Using white light interferometry, the separation distance, the radius of curvature and the interacting force can be in-situ monitored in SFA experiments.[23] Hence, absolute distance referencing and normalization of the measured forces by radius of curvature remain unambiguous. Yet, in SFA experiments, the contact area is macroscopic and typically in the range of hundreds or thousands of $\mu \mathrm{m}^{2}$ which is much larger than that the typical contact area measured in single molecule force spectroscopy [24].

The Atomic Force Microscope (AFM) technique is widely used to measure interaction forces between surfaces at the molecular scale[24]. The schematic of AFM is shown in Figure 1B. In an AFM experiment, a small tip attached to a lever is moved toward a surface. Meanwhile the deflection of the lever and the displacement of the base to which the lever is mounted are measured as shown in Figure 1B. The deflection of the lever is measured using a laser beam reflected into a four quadrant diode and converted into interaction force using Hooke's law. The zero distance (the situation at which the tip is in contact with the sample) in an AFM experiment, however, is assumed by calibration with a hard wall of unknown origin. The exact shape and the 


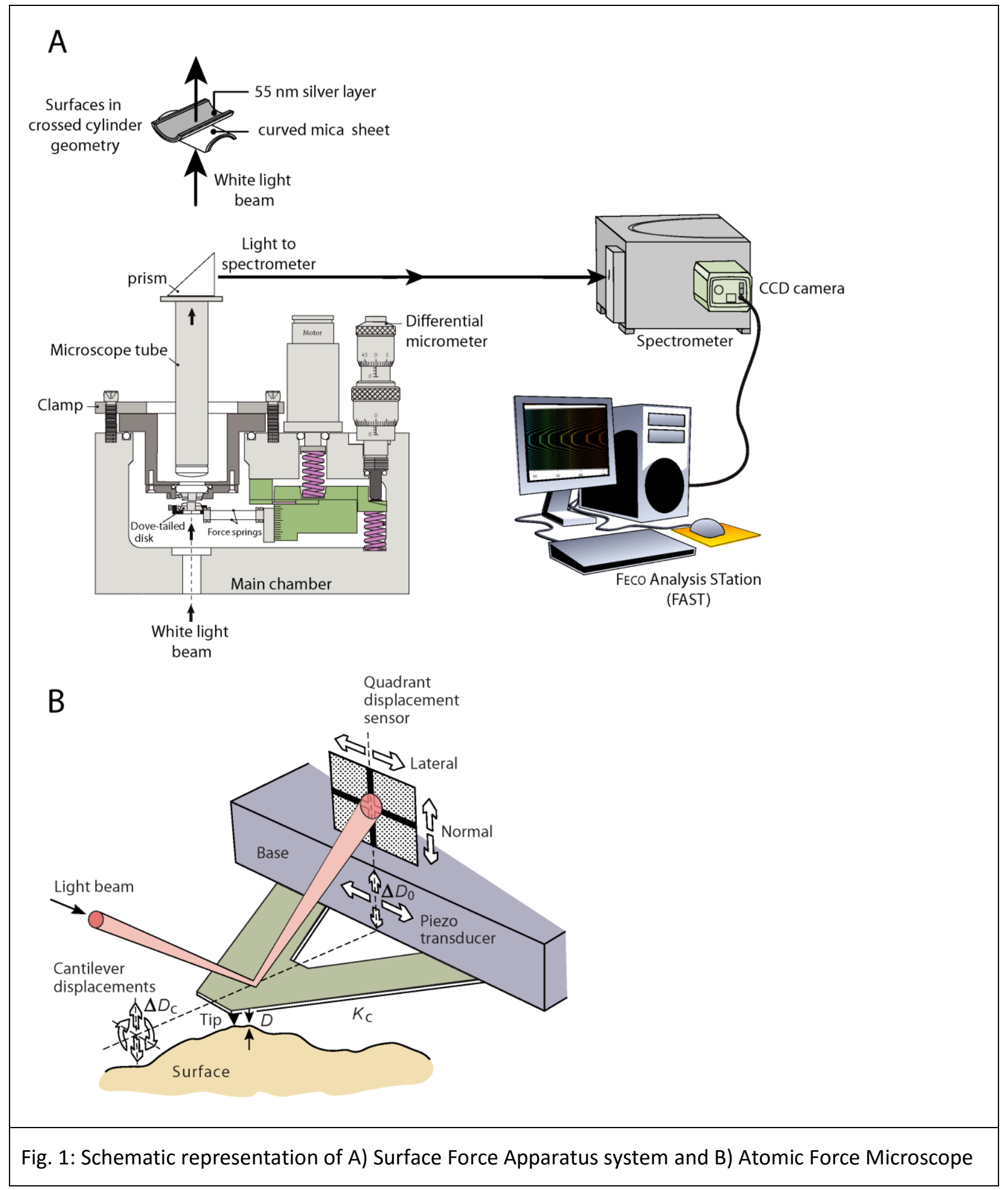


In this report, we review the origin and evolution of the repulsive hydration force in various electrolyte solutions taking into account especially the SFA results, and corroborate the results by simulation and other experimental techniques wherever applicable.

FORCES BETWEEN SOLID SURFACES IN ELECTROLYTE SOLUTION

The very first, accurate and direct measurement of forces between solid mica surfaces immersed in aqueous electrolyte using SFA by Israelachvili and Adams [25] is shown in Figure 2 (the results obtained by Pashley el al. is also combined in Figure 2A). It shows the forces between two charged hydrophilic mica surfaces in $\mathrm{K}^{+}$solutions in the concentration range of 1 to $10^{-5} \mathrm{M}$ at $20^{\circ} \mathrm{C}$ at $\mathrm{pH}$ 6. Figure $2 \mathrm{~A}$ shows pure DLVO interaction below $10^{-3} \mathrm{M}$ and an additional monotonic short range repulsion at or above this concentration for $\mathrm{K}^{+}$ions. Pashley boldly reported the rise of hydration forces only above a certain critical bulk concentration which depends on the electrolyte [26]. Hydration forces become apparent only when hydrated cations adsorbed on mica are prevented from desorbing the surfaces upon approach. Pashley showed that this force is completely absent at $\sim 5 \times 10^{-6} \mathrm{M}$ for $\mathrm{Na}^{+}$and in hydrochloric acid solutions up to $1.2 \times 10^{-3} M$ [27]. Pashley and Israelachvili [28] further reconfirmed the rise of an additional short-range repulsive hydration force only above a certain electrolyte concentration by careful examination of the interaction forces below $2 \mathrm{~nm}$ of separation distance in aqueous $\mathrm{KC} 1$ solutions. The authors showed that the increase of the hydration force occurred both in magnitude and range (attaining 4-5 nm) with the increase of adsorbed ions on the surface. Pashley calculated the magnitude of the hydration force which followed the series $\mathrm{Na}^{+}>\mathrm{Li}^{+}>$ $\mathrm{K}^{+}>\mathrm{Cs}^{+}$using a simple site-binding model to describe the charging behavior of interacting mica surface [29]. The DLVO-regulation theory was subtracted from the total measured force on the assumption that the mica surfaces apparently were fully covered with adsorbed cations. Qualitatively, with the exception of sodium, it seemed that the smaller the ions the stronger is its

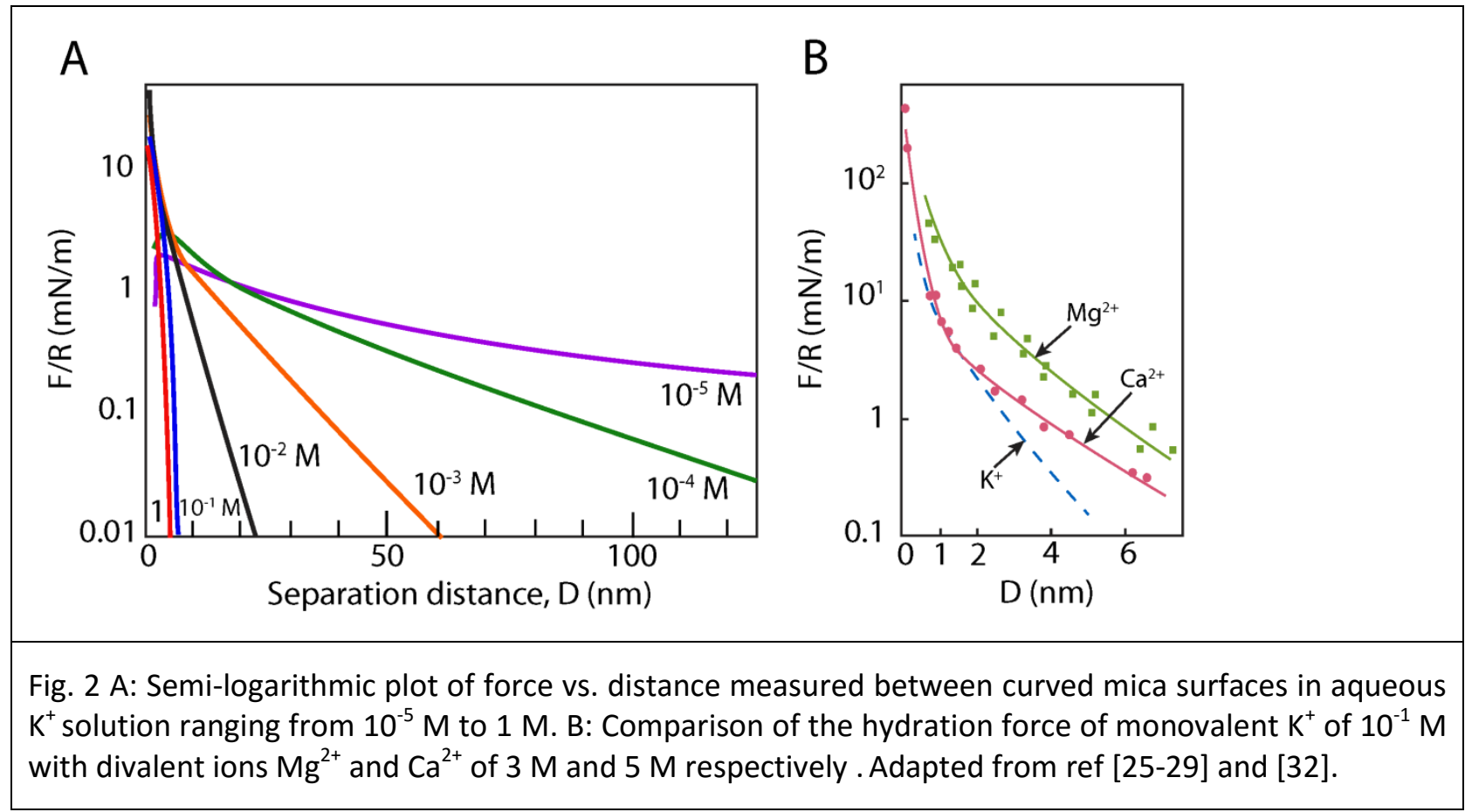


binding capacity to mica. Similar results were observed by Hribar et al. and Goldberg et al. $[30,31]$.

Pashley and Israelachvili [32] also measured the interaction forces between mica surfaces in divalent metal $\left(\mathrm{Mg}^{2+}, \mathrm{Ca}^{2+}, \mathrm{Sr}^{2+}\right.$, and $\left.\mathrm{Ba}^{2+}\right)$ chloride solutions. They found that at concentrations $\geq 1.0 \mathrm{M}$, the divalent cations firmly bind to the interacting mica surfaces giving rise to a repulsive short-range force (see Figure 2B) seemingly due to the residual hydration shells of the bound cations. The authors inferred that the divalent cations were more strongly hydrated than monovalent cations and therefore did not easily shed their hydration layers in order to bind to the mica surface and give rise to hydration force at higher concentrations than monovalent cations do. The magnitude of the hydration force followed the series $\mathrm{Mg}^{2+}>\mathrm{Ca}^{2+}>$ $\mathrm{Li}^{+} \sim \mathrm{Na}^{+}>\mathrm{K}^{+}>\mathrm{Cs}^{+}$. Israelachvili and Adams also observed that the double layer forces were much reduced in $\mathrm{Ca}\left(\mathrm{NO}_{3}\right)_{2}$ and $\mathrm{BaCl}_{2}$ solutions compared to those in $\mathrm{KNO}_{3}$ solutions and were not accurately described by DLVO theory [33]. Using a modified set up of SFA, Rabinovich et al.[34] carried out direct measurements of the interaction forces between gold spheres and crossed quartz filaments in air within the region of distances from 10 to $100 \mathrm{~nm}$. The authors observed deviations from DLVO theory below $5 \mathrm{~nm}$ which they attributed to the influence of structural forces. Ruckenstein et al. [35] suggested that bilayers of charges formed by the adsorption of hydrated ions and that charged bilayer generated a polarization in the neighboring water molecules which propagates into the liquid. The repulsive hydration forces were generated by the overlap of the polarization layers of the two plates.

All the mentioned studies confirmed independently the existence of an interaction force, different from the normal van der Waals and double-layer forces. This force was found to be independent to the type of electrolyte. Therefore it was classified as an additional force and not a modification of the double-layer force. Hence, for the first time, the repulsive forces which later will be defined as hydration forces was introduced. The general inference that is drawn by these early studies is that the force was intrinsically repulsive and decayed monotonically with

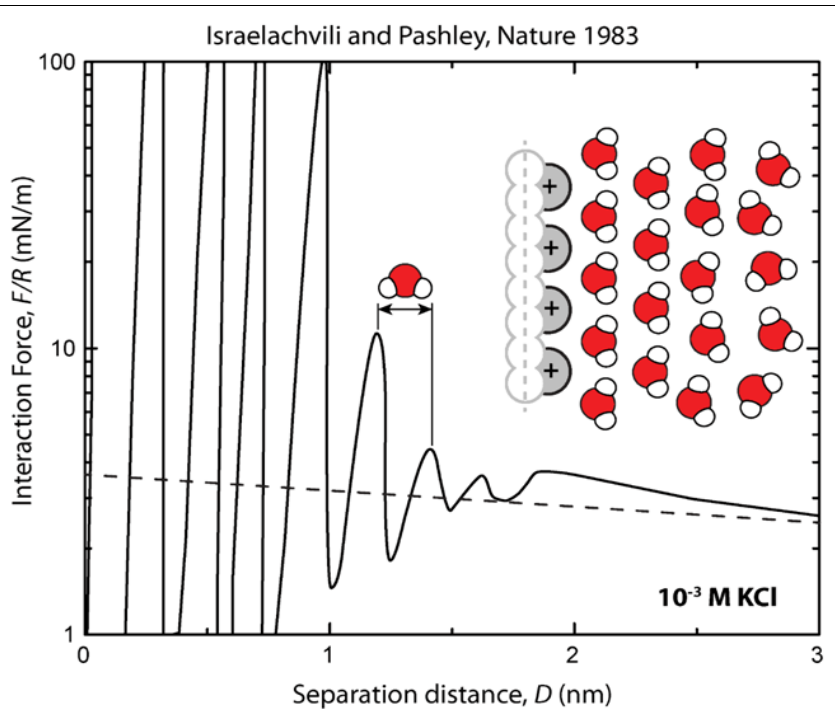

Fig. 3 Force measured between two curved mica surfaces as a function of distance in $10^{-3} \mathrm{M} \mathrm{KCl}$ solution. Adapted from ref [38]. 
distance out to a separation distance of around $5 \mathrm{~nm}$.

On one hand, these results were considered as a breakthrough since the full force law was possible to be determined/verified experimentally but on the other hand, they were contradicting the results of computer simulations $[36,37]$ which were predicting an oscillatory density profile extending several molecular diameters into the liquid. Abraham [36] suspected that the hydration force would probably arise between hydrophilic surfaces, such as silica and mica, since strongly H-bonding surface groups modify the H-bonding network of nearby liquid water molecules.

In 1983 Israelachvili and Pashley[38] meticulously measured the hydration force in $10^{-3}$ $\mathrm{M} \mathrm{KCl}$ solution of $\mathrm{pH} 5.5$ between molecularly smooth mica surfaces. The authors reported that the hydration force was not monotonic once the separation distance was smaller or equal to $20 \AA$. The hydration force was oscillatory having a minima and maxima of periodicity $2.5 \pm 0.3 \AA$, roughly the diameter of a water molecule (Fig. 3). The schematic in Figure 3 shows that the adsorption of ions on the mica surface orders water molecule at the surface. Using atomic force microscopy(AFM) and computer simulations, Ricci et al. [39] recently showed that cations

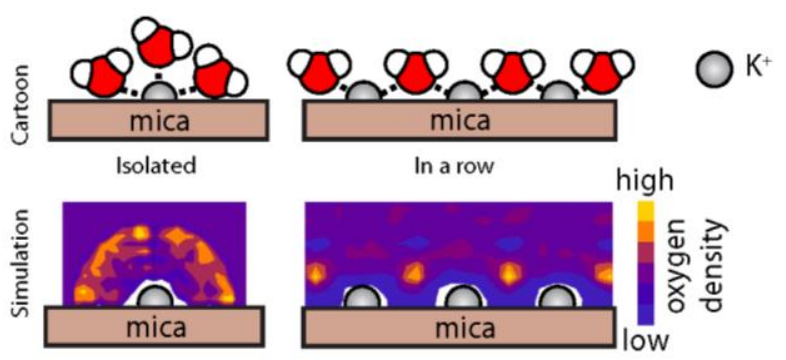

Fig. 4: The schematic representation of cation/s $\left(\mathrm{K}^{+}\right)$being randomly adsorbed (a) and adsorbed forming a row on mica (b). Similarly $c$ and $d$ represents the density of oxygen atoms of the water molecules. In the case of single ion, the value of oxygen density is the radial average around $\mathrm{K}^{+}$ions. In the latter case, the average oxygen density is obtained by considering a rectangle of $0.5 \mathrm{~nm}$ width aligned with the row of the ions and centered on them. Adapted from ref [39].

adsorbed on the surface of mica could induce ordered water layers at the surface of homogeneous solids in aqueous solutions as shown in Figure 4. Similar results were reported in a recent study using molecular dynamics simulations between two mica surfaces in an aqueous $\mathrm{KCl}$ electrolyte solution by Leng et al. [40]. Urbic et al. [41] also came to the same conclusion in a simulation study. The authors used the Mercedes-Benz (MB) model of water, in NVT and $\mu$ VT Monte Carlo computer simulations and observed oscillations in the forces between inert plates, due to water structure, even at plates separation of 5-10 water diameters. Cherepanov et al. [42] showed the increase of the hydration repulsion with ionic strength by molecular dynamics simulation. Henderson et al. [43] reported 7 force oscillations below $2 \mathrm{~nm}$ between spheres brought in contact using a non-continuum molecular theory. Using grand canonical moleculardynamics simulations. Li et al. [44] reported oscillatory solvation forces between hydrophilic mica and glass surfaces. Fenter et al. [45, 46] studied the mica (001)-water interface under ambient conditions using High-resolution x-ray reflectivity. The results revealed density 
oscillations of the water oxygen atoms in the normal direction to the surface, providing evidence of interfacial water ordering. The spacing between neighboring water layers in the near-surface region, were approximately $2.5(2)-2.7(2) \AA$, close to the size of a water molecule. The density oscillations extended to about $10 \AA$ above the surface and did not strictly maintain a constant periodicity. The authors came to the conclusion that the primary hydration layer was followed by a weakly modulated hydration structure that extended more than $1 \mathrm{~nm}$ above the surface.

The described experimental and simulation results show that for monovalent ions the hydration force between hard surfaces appears only above a critical ion concentration and is not only monotonically repulsive but has an oscillatory component superimposed to it.

Attempts have been made to measure the forces between surfaces other than mica. Horn et al. [47] measured the monotonic short-range repulsive force devoid of oscillatory component in $\mathrm{NaCl}$ solutions between silica surfaces. The measured force was found insensitive to the ionic conditions making it apparently intrinsic to the surfaces. Similar forces have been measured between silica surfaces by Vigil et al. [48], Ducker et al. [49], between mica surfaces by Shubin et al. [50], between (0001) sapphire surfaces by Drucker et al. [51], and between glass fibers by Rabinovich et al. [34] using different techniques. According to Iler [52, 53], silica is amorphous and its negative surface charges are located at the ends of short silica hairs protruding a few angstroms from the surfaces which shifts the Outer Helmholtz Plane farther out than the physical solid-liquid interface (the van der Waals plane) causing the DLVO interaction to be more repulsive. A similar effect is observed for finite sized counterions adsorbed on mica surfaces [54, 55]. Chapel [56] measured forces between two pyrogenic silica sheets immersed in monovalent electrolytes $(\mathrm{CsCl}, \mathrm{KC} 1, \mathrm{NaC} 1, \mathrm{LiC} 1)$. Contrary to the previous results, his results showed that the strength and the range of the hydration force decrease with increasing the degree of hydration of the counter ion. This is opposite to the behavior of mica for which adsorbed counter ions have been reported to generate a hydration repulsion. The effects of counter ions on hydration forces, weakening for silica and enhancing for mica, show that the origin of the short-range interaction is not unique.

The existence of hydration force was evidenced by several researchers using various modes of AFM [49, 57-67]. As postulated by Israelachvili et al.[21], experimentally shown by Atkins et al. and Zeng et al.[68, 69], and theoretically shown by Yang et al. [70]; a tip roughness greater than the molecular diameter of the water molecule plays an important role in smearing out the oscillatory component of the hydration force. Adsorbed chemical compounds can also alter significantly the stability of the hydration layer as recently shown by Akrami et al. [71]. Direct in situ characterization of the AFM tip or of any interacting surface cannot be performed till date which limits our understanding of the role of surface roughness or chemistry in the appearance or disappearance of hydration forces. For more details, readers are suggested to consider refs $[68,72-77]$.

As discussed, the simulation studies have always been leading showing the layered density profile [36, 78-80] analogous to the hydration shell and/or evidences of interfacial layering near interfaces [81-93] for various types of substrates. Layered density profile were also experimentally shown under potential control [94-97]. The recent advancement of X-ray techniques [98-103] have enabled atomic-scale studies of interfaces. Liquid density oscillations near solid surfaces have been observed in many systems [104-108]. Neutron scattering 
measurements have also demonstrated the existence of structured fluid layers under confinement 209 [88, 89], [109-112]. Mineral-water interface using X ray has been studied by Fenter et al. [46, 210 113-118]. For details mineral-water interfacial structures revealed by synchrotron X-ray 211 scattering refer to references [45, 119]

212 Thus from above close examination of the studies, both experimental and theoretical, we 213 can draw the following conclusions:

1. Above the critical concentration, the adsorption of cation on the mica surface is the cause of the repulsive hydration force and the hydration force is not only monotonically repulsive but an additional oscillatory component is superimposed to it below $2 \mathrm{~nm}$ and its periodicity is equal to the mean molecular diameter of water.

2. The critical concentration depends on the type of the electrolyte. The critical concentration for divalent metal ion is always higher than that for the monovalent metal ion solution.

3. The magnitude of the oscillatory force component depends on the extent of adsorption of cation on the surface which in turn depends on the concentration of the solution. The more the cations adsorbed the strong is the hydration force.

4. Surface roughness plays an important role in smearing out the oscillatory component of the hydration forces. 
229

230

231

232

233

234

235

236

237

238

239

240

241

242

243

244

245

246

247

Lipid bilayers have been extensively studied in the past 50 years. Lipid bilayer are formed by the self assembly, in water, of lipid molecules into a bilayer structure through hydrophobic interactions between hydrocarbon tails of the molecules. As a consequence, the interfacial properties of a bilayer differs significantly from those of solid surfaces mostly due to the inherent mobility of the lipid molecules forming the bilayers. As we will see, such mobility dramatically modulates the interaction of the bilayer with water molecules, solvated ions and with other bilayers as well. In what follows, we describe the recent efforts that have been made towards a better understanding of such interactions and the future directions of research that these recent results are offering.

\section{The water-bilayer interface}

The structure of the water bilayer interface has been studied extensively using spectroscopic techniques such as Nuclear Magnetic Resonance (NMR) and Infrared (IR spectroscopy).[120] Early studies performed using NMR and molecular dynamic simulation (MDS) focused on identifying the number of molecules necessary to form the hydration shell of lipids. It is commonly accepted that this number varies between 17-22 water molecules depending on the lipid head group, lipid area [121] and availability of ions or solutes and temperature (see Figure 5).[122] More recent simulations have suggested that the interfacial water can mostly be classified into three categories which are $[123,124]$ (i) buried water located close to the carbonyl groups, (ii) first external hydration shell near the phosphorylcholine groups and (iii)

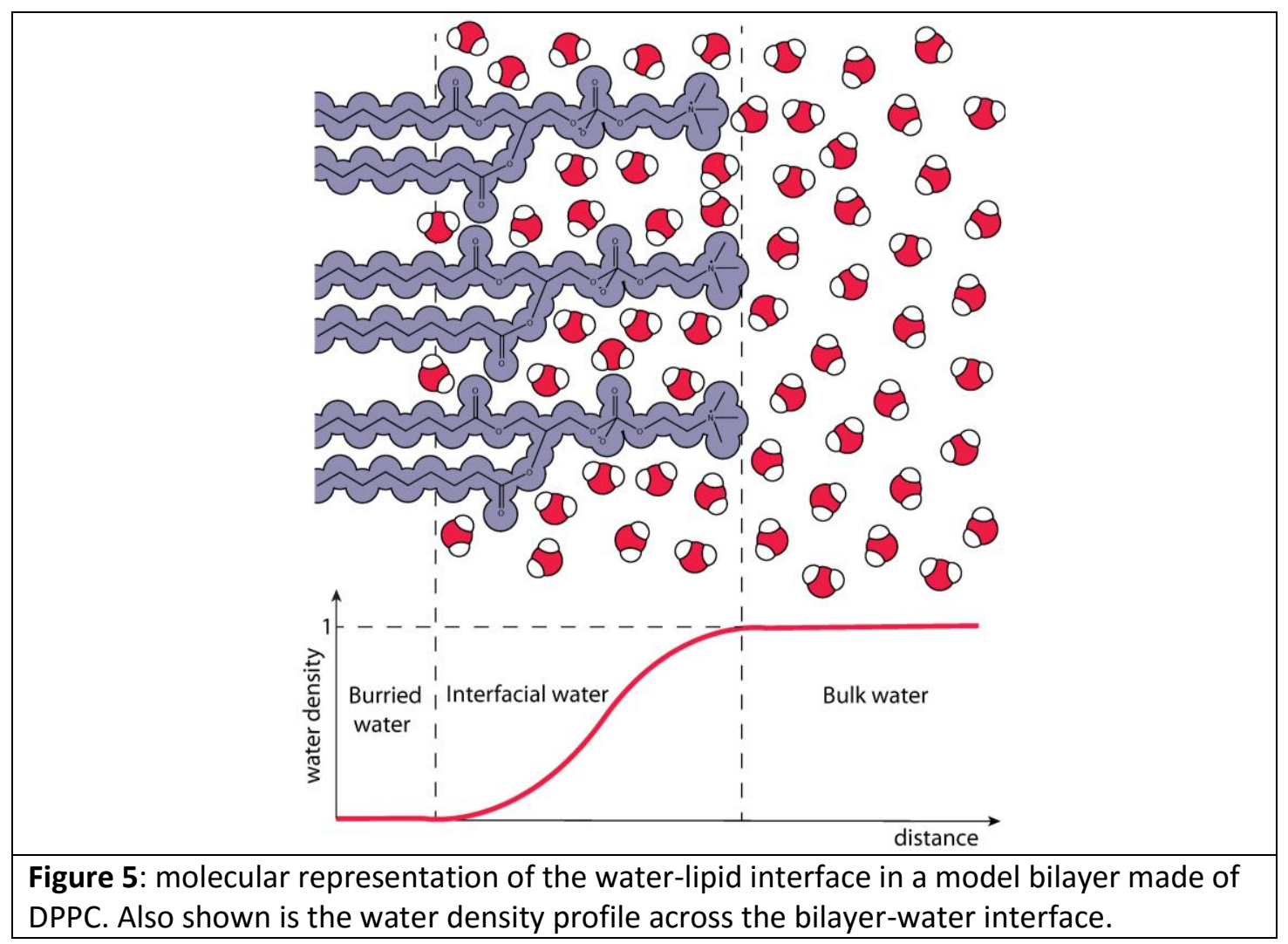


experimentally, there are clear experimental evidences of strongly bound water molecules to lipid head groups.

Water distribution around lipid head group can be easily disturbed by external factor. In particular, presence of ions, especially cations have been shown experimentally to alter lipid molecular area and diffusivity. Since $\mathrm{Na}^{+}$is the most physiologically abundant, its interaction with lipid bilayers has been extensively studied, theoretically and experimentally. For example Fluorescence Correlation Spectroscopy (FCS) has been used to characterize POPC self-diffusion coefficient in presence of varying concentration of $\mathrm{NaCl}$ at different temperatures. The study shows that independent to the fluorescent marker used, the augment in concentration of salt tend to decrease the diffusion of POPC molecules.[125] Simulation studies confirmed this observation and revealed that tight coordination complexes involving one $\mathrm{Na}^{+}$, three lipid molecules and 1-2 water molecules could be formed. Similar results were reported with mixed lipid bilayers.[126]

The binding of other types of species such as organic molecules have also been reported to strongly affect the behavior of water molecules near the bilayer interface. For example, dimethyl sulfoxide (DMSO) has been widely studied for its ability to preserve cellular membrane during cryo-preservation. Such important property was recently explained using PFG NMR and the SFA.[127]

\section{Interaction forces between lipid bilayers}

The interaction forces acting between apposing lipid bilayers include the following: [52]

(i) van der Waals interactions forces between the bilayer hydrocarbons layer across water,

(ii) hydration forces due to the expulsion of water molecules upon compression of the bilayers,

(iii) the hydrophobic interaction between lipid tails across water,

(iv) the protrusion interaction forces due to the restriction of lipids position fluctuations along the direction of compression,

(v) the lipid head group overlap involving conformational change of the head groups upon compression,

(vi) the undulation forces due to bending fluctuations of the bilayers and

(vii) the electrostatic interaction forces due to bilayer surface charging.

Each of these contributions have been studied both experimentally and theoretically. Mathematical expressions of these interactions are provided in Table 1 and the reader is referred to the original references for further details of their derivation.

Interaction forces between lipid bilayers or lipid membranes have been measured using a variety of techniques. The two main techniques have been the osmotic pressure technique (OP) and the SFA. As can be seen in Figure 6A and B, both measurement techniques give similar results on single component lipid bilayers and multi-component lipid bilayers/membranes. Important differences should also be noticed: when using the OP technique, the thickness of the lipid bilayers increases (the lipid molecular area decreases) as the applied pressure increases; while in 
the SFA measurement, the lipid bilayer becomes thinner (the molecular area increases) at the contact area due to lateral diffusion of lipids during the compression. These important differences explain why hemifusion/fusion of bilayers is rarely reported using the OP technique and can be easily observed using the SFA.

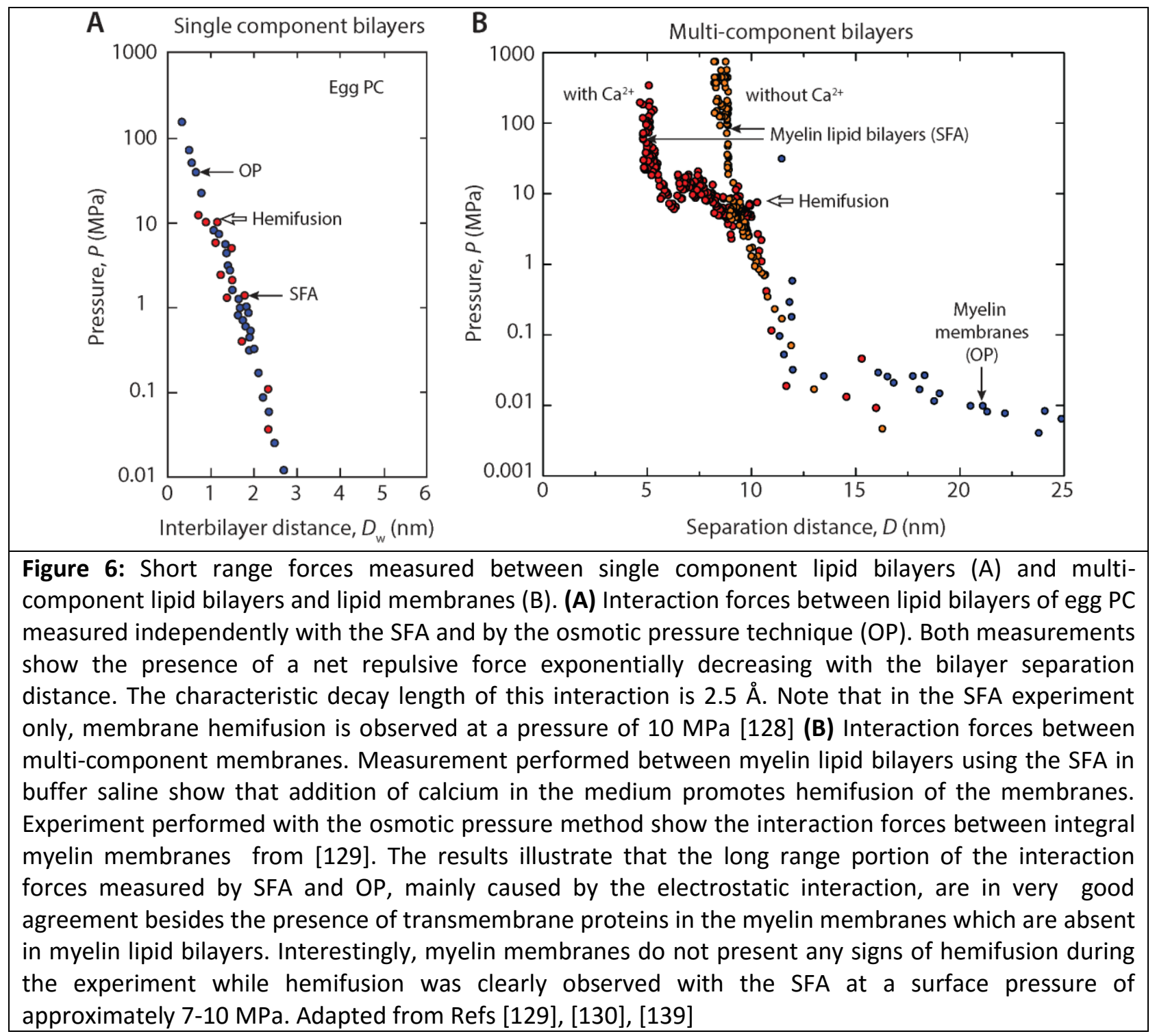


Table 1: Interaction forces commonly encountered at lipid bilayers and at solid surfaces

\begin{tabular}{|c|c|c|c|}
\hline Contribution & Equation & & Reference \\
\hline \multirow{2}{*}{$\begin{array}{l}\text { Head group } \\
\text { overlap }\end{array}$} & $P(D)=\frac{\mathrm{kT}}{\mathrm{s}^{3}}\left[\left(\frac{2 L}{D}\right)^{9 / 4}-\left(\frac{D}{2 L}\right)^{3 / 4}\right]$ for $\mathrm{D}<2 \mathrm{~L}$ & (Eq. 1) & {$[130,131]$} \\
\hline & $P(D)=\frac{100}{\mathrm{~s}^{3}} \mathrm{kTe}^{-\pi \mathrm{D} / \mathrm{L}}$ for $0.4 \mathrm{~L}<\mathrm{D}<1.8 \mathrm{~L}$ & (Eq. 2) & \\
\hline Undulation & $P(D)=\frac{3 \pi^{2}(\mathrm{kT})^{2}}{64 \mathrm{k}_{\mathrm{b}} \mathrm{D}^{3}}$ & (Eq. 3) & {$[132]$} \\
\hline $\begin{array}{l}\text { Hydrophobic } \\
\text { interaction }\end{array}$ & $\begin{array}{l}P(D)=-\frac{\mathrm{e}^{-\mathrm{D} / \mathrm{D}_{\mathrm{H}}}}{\mathrm{D}_{\mathrm{H}}}\left[\left(1-\left(1-\mathrm{e}^{-\mathrm{D} / \mathrm{D}_{\mathrm{H}}}\right)^{1 / 2}\right)+\right. \\
\left.\frac{1}{2}\left(\mathrm{e}^{-\mathrm{D} / \mathrm{D}_{\mathrm{H}}}\left(1-\mathrm{e}^{-\mathrm{D} / \mathrm{D}_{\mathrm{H}}}\right)^{-1 / 2}\right)\right]\end{array}$ & (Eq. 4) & [133-135] \\
\hline $\begin{array}{l}\text { Protrusion } \\
\text { interaction }\end{array}$ & $\begin{array}{l}P(D)=2.7(\Gamma \mathrm{kT} / \lambda) \mathrm{e}^{-\mathrm{D} / \lambda} \\
\lambda=k T / \pi \delta \gamma_{i}\end{array}$ & (Eq. 5) & {$[136,137]$} \\
\hline van der Waals & $P(D)=\frac{-\mathrm{A}}{6 \pi \mathrm{D}^{3}}$ & (Eq. 6) & {$[52]$} \\
\hline Electrostatic & $P(D)=64 \mathrm{kT} \rho_{\infty} \tanh ^{2}\left(\mathrm{ze} \psi_{0} / 4 k T\right) \mathrm{e}^{-\mathrm{D} / \lambda_{\mathrm{D}}}$ & (Eq. 7) & {$[52]$} \\
\hline Hydration & $P(D)=\mathrm{W}_{0} \mathrm{e}^{-\mathrm{D} / \mathrm{D}_{\mathrm{H}}}$ & (Eq. 8) & {$[52]$} \\
\hline $\begin{array}{l}\text { Oscillatory } \\
\text { (structural) }\end{array}$ & $P(D) \approx W_{0} / \sigma \mathrm{e}^{-\mathrm{D} / \sigma}\left[2 \pi \sin \frac{2 \pi \mathrm{D}}{\sigma}-1\right]$ & (Eq. 9) & {$[52]$} \\
\hline
\end{tabular}

It is important to note that all these interaction forces originates from very distinct causes and therefore exhibit very different magnitudes and act over very different length scales. As a consequence, the separation distance $D$ between the bilayer does not have the same origin point or (operational plane) in each contribution.[52] For example, the plane of origin of the electrostatic interaction should be located at the position where surface charges are located which might be quite different from the origin plane of the van der Waals interaction. Shifting of the plane of origin of an interaction potential is usually done by replacing the separation distance $D$ 302 by $D-D_{0}$ where $\mathrm{D}_{0}$ is the shifting distance.

All the interactions presented in Table 1 do not have to be necessarily considered in every situation. For example in a physiological fluid or in a buffer saline solution containing salt concentrations of typically $150 \mathrm{mM}$ (1:1 electrolyte), the van der Waals interaction is usually found to be negligible compared to other contributions. The presence of free ions in the medium 
screens the zero-frequency term of the van der Waals interaction over a distance equals to the Debye screening length (roughly $1 \mathrm{~nm}$ at physiological conditions).

The hydrophobic interaction is usually considered to play a minor role in the total interaction between bilayers mainly because it is generally counterbalanced by other contributions such as the electrostatic or the head group overlap force. Hydrophobic forces should only be considered when hemifusion/fusion of the bilayers occurs. As the bilayers are compressed, thinning will start to occur and will favor the exposure of the hidden hydrophobic core of the bilayer to the aqueous medium. It is clear then that appearance of the hydrophobic interaction between facing bilayers depends on lipids molecular coverage and its variation with confinement. Donaldson et al. [133] provided a simple expression for the molecular area of elastic lipid bilayers in presence of the hydrophobic interaction:

$$
a(D)=\mathrm{a}_{0}\left(1-\mathrm{e}^{-\mathrm{D} / \mathrm{D}_{\mathrm{H}}}\right)^{-1 / 2}
$$

Using the expression of the hydrophobic interaction together with the electrostatic and steric contributions, Banquy et al. described the interaction forces between two model myelin lipid bilayers immersed in buffered saline solution.[138] The authors showed that consideration of the hydrophobic interaction allowed to predict the onset of hemifusion of the bilayers which occurred when the lipid molecular area increased by almost 30\% (see Figure 7A). The critical molecular area at which hemifusion appears, i.e. the point where the hydrophobic interaction counterbalances all other repulsive interactions, is strongly dependent on the cohesive strength of the bilayers and the lipid molecular area.

Figure 7B presents a situation where a photosensitive bilayer was deposited on mica by the vesicle deposition method. When compressed, the bilayers hemifused at a critical molecular area which was $16 \%$ higher than the equilibrium value. When illuminated under UV light, the surfactant molecules changed conformation and became more hydrophilic which dramatically expanded both the interfacial energy and the molecular area of the molecules at rest. Thus, the critical molecular area at hemifusion was found to be $25 \%$ higher than the value at equilibrium. 

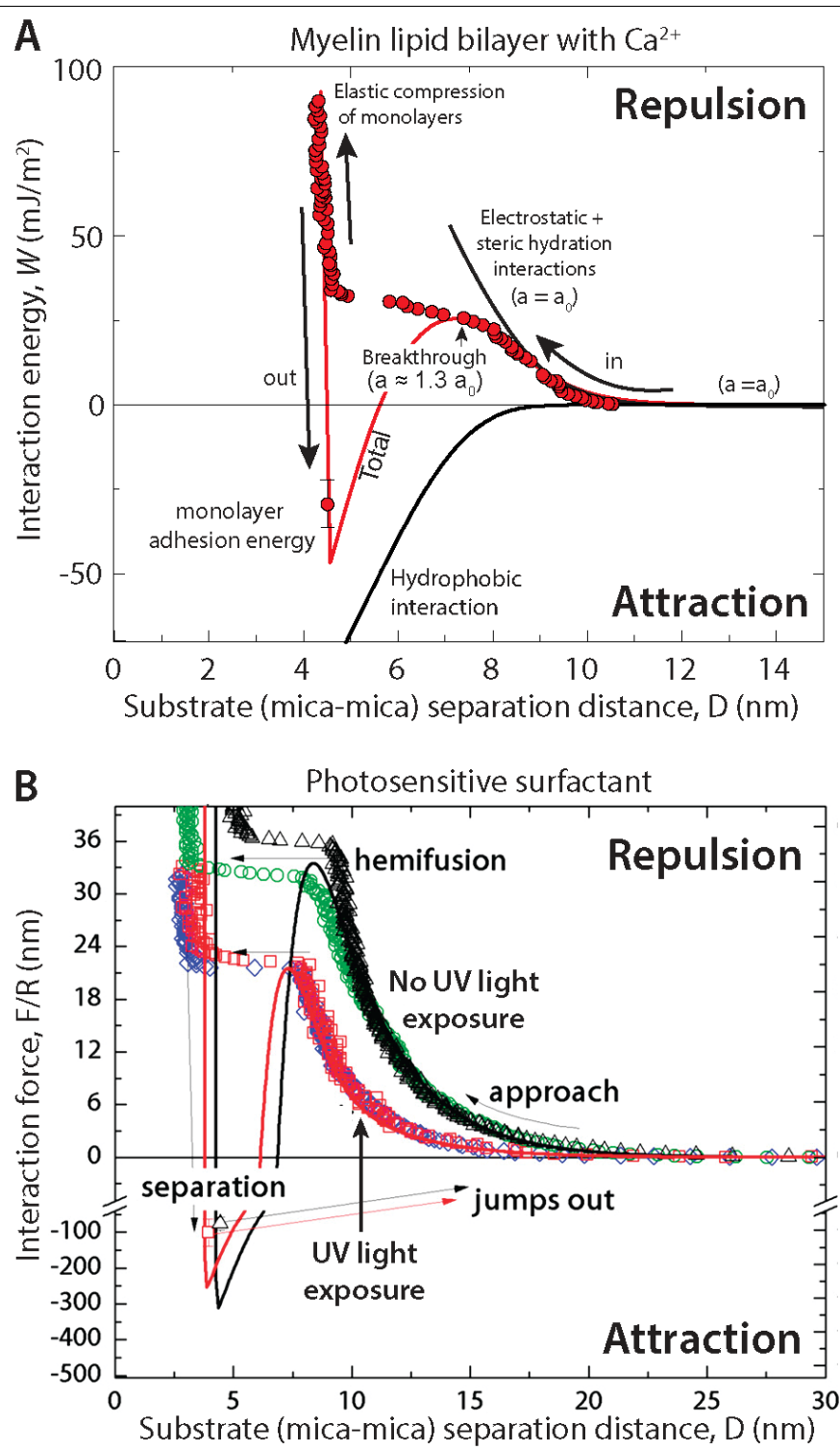

Figure 7: (A) Interaction energy between two myelin lipid bilayers in presence of $2 \mathrm{mM} \mathrm{Ca}^{2+}$ ions. Calcium ions facilitate the hemifusion of the bilayers as denoted by a breakthrough instability in the interaction energy profile. Such instability is governed by the hydrophobic interaction between the hydrocarbon tails of the lipids that are being exposed during compression and thinning of the bilayers. Adapted from [139] (B) Interaction force measured between photosensitive surfactant bilayers. In this system the hydrophobic force was modulated by the exposure of the light sensitive hydrocarbon tails to UV light leading to different energetic barrier to hemifusion. Adapted from [139]

The undulation interaction has by far the longest range and is comparable in magnitude to the van der Waals interaction. The magnitude of this interaction can be strongly by different external parameters. For example, lipid bilayer supported on a solid substrate will almost no undulations. As shown in Eq. 3, undulation forces are also extremely sensitive to temperature which can be useful in experimental settings where fine tuning of this interaction is required. Ions have also an important impact on undulation forces. Free ions in solution can either adsorb on lipid 
membranes, alter head group dissociation degree or their hydration level. All these factors will impact directly or indirectly the bending modulus of the bilayer and therefore the undulation forces. For low salt concentration (or large surface charges),[140] derived the following expression for the bending modulus of symmetrical bilayers:

$$
k_{b}=\frac{\varepsilon \lambda_{\mathrm{D}}}{\pi}\left(\frac{\mathrm{kT}}{\mathrm{e}}\right)^{2}
$$

Considering that $\lambda_{D}=0.304 / \sqrt{C}$ for a $1: 1$ electrolyte at room temperature, simplification of Eq. 9 leads to $P \propto \sqrt{C}$ which results in a weak dependence on salt concentration. In the high salt regime, the expression of the bending modulus becomes:

$$
k_{b}=\frac{3 \pi\left(\lambda_{\mathrm{D}}\right)^{3} \sigma^{2}}{\varepsilon}
$$

where $\sigma$ is the surface charge and $\varepsilon$ the dielectric constant of the medium. Using the Grahame equation for a 1:1 electrolyte, the expression for $\sigma$ becomes $\sigma=\sqrt{8 \varepsilon \varepsilon_{0} \mathrm{kT}} \sinh \left(\mathrm{e} \psi_{0} / \mathrm{kT}\right) \sqrt{\mathrm{C}}$ which leads to the same weak dependence for the interaction pressure $P \propto \sqrt{C}$.

The head group overlap and protrusion forces are usually difficult to distinguish from each other and are often termed "steric hydration" forces. The protrusion force is related to the hydrophilicity of the bilayer while the head group overlap force is more related to the hydration of the head groups. In both cases, it is important to remember that these forces do not originate from water structuring at the bilayer-water interface. Since both interactions exhibit an exponential decay (see Eq. 2 and 5), comparison of their characteristic decay length should in principles provide useful insights into the contribution that dominates the total interaction. A back of the envelope calculation already provides good estimates of the expected values of these two parameters. Considering the interfacial tension between water and hydrocarbon to be 50 $\mathrm{mJ} / \mathrm{m}^{2}$ and the cross sectional radius of the hydrocarbon chain to be close to $0.2 \mathrm{~nm}$, we found using Eq. $5, \lambda=0.13 \mathrm{~nm}$. On the other hand, the size of a phosphatidyl head group is expected to be close to $0.8 \mathrm{~nm}$ giving (Eq. 2) $\lambda=0.25 \mathrm{~nm}$. Since these two decay lengths are comparable, it is a priori difficult to identify the dominant one.

In a recent study by Schrader et al. [141], the authors characterized the effect of DMSO, in water, on the interactions between bilayers. The authors elegantly demonstrated that by increasing the concentration of DMSO in water from $0 \%$ to $7.5 \%$, the decay length of the interaction force markedly decreased from 0.2 to $0.15 \mathrm{~nm}$ (see Fig. 9). Since the interfacial tension between water/DMSO and hydrocarbon decreases with increasing the DMSO content, it is clear that the measured change in decay length could not be accounted by the presence of protruding forces. However, using NMR diffusion measurements, the authors showed that the change in decay length observed in presence of higher concentrations of DMSO was correlated with a change in hydrodynamic radius of the head group ionic moieties. The authors concluded that the interaction forces between DPPC bilayers at separation distance lower than $2 \mathrm{~nm}$ were dominated by the head group overlap force which is tuned by the competition between DMSO and water molecules to interact with the lipid head group. 


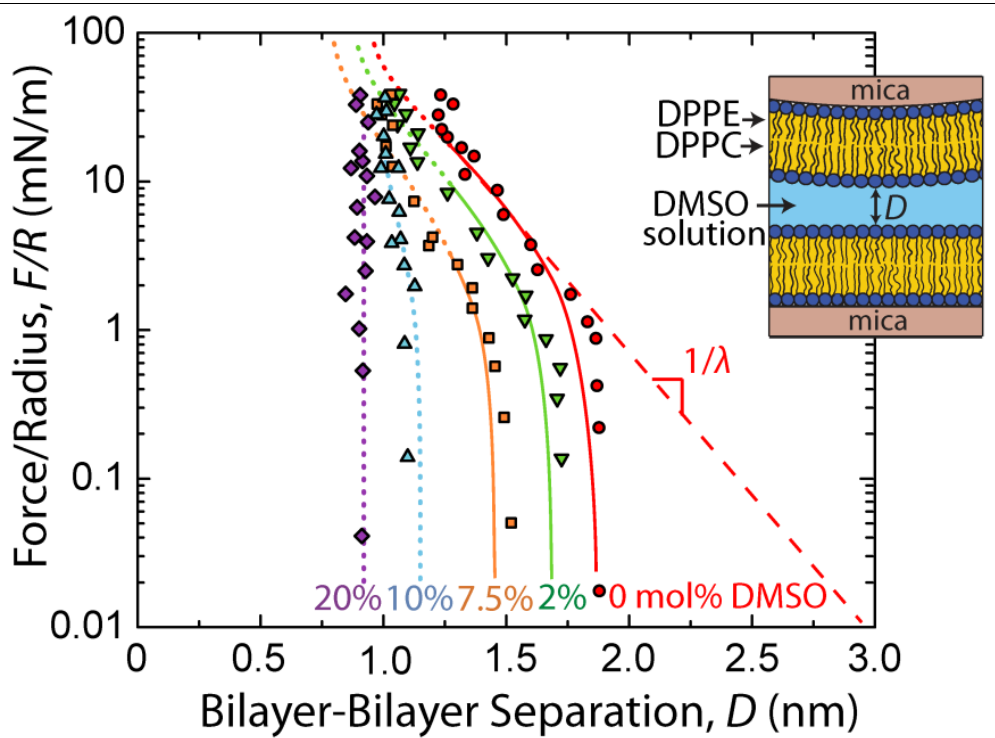

Fig.8 Short range interaction forces measured between DPPC lipid layers in presence of a water-DMSO solution. Measurements show a decrease of the range and the decay of the interaction forces which is a characteristic signature of a conformational change of the lipid head group mediated by DMSO. Adapted from [142].

These recent and previous studies [142-144] underscore the important fact that the so called "steric hydration" is actually an steric-entropic force similar to the polymer-brush interaction described by Alexander and deGennes. The role of water is solely to modulate the hydration, and therefore the conformation, of the lipid head groups. The effect of water on the degree of hydration of a lipid head group is a solvation effect which in term of interaction forces will manifests itself as a shift in the interaction, while the effect on the lipid head group will change the decay length and the range of the interaction. The understanding of these subtle effects provides a powerful framework to study the interaction of a large number of solute molecules such as ions or small molecules such as peptides interacting directly or indirectly with the surface of lipid bilayers.

In light of this review of the literature on the measurements of the hydration interaction we can highlight a few similarities and differences when comparing the force laws obtained with solid and soft (fluid) surfaces. In terms of similarities, for both systems, the hydration force exhibits a monotonically decaying component whose decay length varies between 0.1 and $1 \mathrm{~nm}$. The origin of this component is quite different in both systems: for solid surfaces it involves water molecules bound to the surfaces either directly or via strongly adsorbed ions, while for soft lipid bilayers it is related to the lipid head-group conformation and level of hydration. A clear evidence that origin of the hydration force in both systems is different can be appreciated in the fact that the hydration force between solid surfaces appears only above a critical salt concentration which is not the case for soft bilayers. Hard atomically flat surfaces are the only system exhibiting an oscillatory component of the hydration superimposed to the monotically decaying component. The appearance of this component is in part related to water conformational polarization induced by the presence of ions on the surface. 
403 Finally, it is important to remind that the appearance of the hydration force has been quite often 404 associated mainly to the ordering water molecules on the surfaces. Hydration forces rise from the 405 overlapping and therefore rearrangement of this ordering of water molecules. There is obviously 406 a connection between ordering/structuring of water and the hydration force, but both phenomena 407 should be always carefully distinguished.

408

409

410 
[1] Derjaguin BV, Landau L. Acta Phys Chim URSS 1941;14. 1948.

[3] Israelachvili JN. Measurement of hydration forces between macroscopic surfaces. Chem Scr. 1985;25:7-14.

[4] Frens G, Overbeek JTG. J Colloid Interface Sci. 1972;38.

[5] Overbeek JTG. J Colloid Interface Sci. 1977;58.

[6] Leneveu DM, Rand RP, Parsegian VA. Nature. 1976;259:601.

[7] Parsegian VA, Fuller N, Rand RP. Proc Natl Acad Sci USA. 1979;76:2750.

[8] Lis LJ, McAlsiter M, Fuller N, Rand RP, Parsegain VA. Biophys J. 1982;37:657.

[9] van Olpen H. An introduction to clay colloidal chemistry. Wiley: New York; 1977.

[10] Viani BE, Low PF, Roth CBJ. J Colloid Interface Sci. 1983;96:229.

[11] Rau DR, Lee BK, Parsegain VA. Proc Natl Acad Sci USA. 1984;81:2621.

[12] Tabor D, Winterton RHS. Proc R Soc Lond Ser A. 1969;312:435.

[13] Israelachvili JN, Tabor D. Proc R Soc Lond Ser A. 1972;331:19.

[14] Israelachvili JN, Tabor D. Prog Surf Membrane Sci. 1973;7:1.

[15] Huang J, Yan B, Faghihnejad A, Xu H, Zeng H. Understanding nanorheology and surface forces of confined thin films. Korea-Australia Rheology Journal. 2014;26:3-14.

[16] Banquy X, Lee DW, Das S, Hogan J, Israelachvili JN. Shear-Induced Aggregation of Mammalian Synovial Fluid Components under Boundary Lubrication Conditions. Advanced Functional Materials. 2014;24:3152-61.

[17] Baimpos T, Shrestha BR, Hu Q, Genchev G, Valtiner M. Real-time multiple beam interferometry reveals complex deformations of metal-organic-framework crystals upon humidity adsorption/desorption. J Phys Chem C. 2015;119:16769-76.

[18] Shrestha BR, Baimpos T, Raman S, Valtiner M. Angstrom-resolved real-time dissection of electrochemically active noble metal interface. ACS Nano. 2014;8.

[19] Shrestha BR, Hu Q, Baimpos T, Kristiansen K, Israelachvili JN, Valtiner M. Real-time monitoring of aluminum crevice corrosion and its inhibition by vanadates with multiple beam interferometry in a surface forces apparatus. J Electrochem Soc. 2015;162:C327-C32. [20] Heo J, Kang T, Jang SG, Hwang DS, Spruell JM, Killops KL, et al. Improved Performance of Protected Catecholic Polysiloxanes for Bioinspired Wet Adhesion to Surface Oxides. J Am Chem Soc. 2012;134:20139-45.

[21] Israelachvili JN, McGuiggan PM. Forces between surfaces in liquids. Proc R Soc Lond Ser A. 1988;241:795-800.

[22] Israelachvili JN, Min Y, Akbulut M, Alig A, Carver G, Greene W, et al. Recent advances in the surface forces apparatus (SFA) technique. Rep Prog Phys. 2010;73:036601.

[23] Kang T, Amir RJ, Khan A, Ohshimizu K, Hunt JN, Sivanandan K, et al. Facile access to internally functionalized dendrimers through efficient and orthogonal click reactions. Chem Comm. 2010;46:1556-8.

[24] Raman S, Utzig T, Baimpos T, Shrestha BR, Valtiner M. Deciphering the scaling of singlemolecule interactions using Jarzynski's equality. Nature Communications. 2014;5. [25] Israelachvili JN, Adams GE. Direct measurement of long range forces between two mica surfaces in aqueous $\mathrm{KNO}_{3}$ solution. Nature. 1976;262. 
[26] Pashley RM. DLVO and hydration forces between mica surfaces in $\mathrm{Li}^{+}, \mathrm{Na}^{+}, \mathrm{K}^{+}$, and $\mathrm{Cs}^{+}$ electrolyte solutions: A correlation of double-layer and hydration forces with surface cation exchange properties J Colloid Interface Sci. 1981;83:531-46.

[27] Pashley RM. Hydration forces between mica surfaces in aqueous electrolyte solutions. J Colloid Interface Sci. 1981;80:153-62.

[28] Pashley RM, Israelachvili JN. Molecular layering of water in thin-films between mica surfaces and its relation to hydration forces. J Colloid Interface Sci. 1984;101:511-23. [29] Pashley RM. Hydration forces between mica surfaces in electrolyte solutions. J Colloid Interface Sci. 1982;16:57-62.

[30] Hribar B, Southall NT, Vlachy V, Dill KA. How ions affect the structure of water. J Am Chem Soc. 2002;124:12302-11.

[31] Goldberg R, Chai L, Perkin S, Kampf N, Klein J. Breakdown of hydration repulsion between charged surfaces in aqueous $\mathrm{Cs}^{+}$solutions. Phys Chem Chem Phys. 2008;10:4939-45. [32] Pashley RM, Israelachvili JN. DLVO and hydration forces between mica surfaces in $\mathrm{Mg}^{2+}$, $\mathrm{Ca}^{2+}, \mathrm{Sr}^{2+}$, and $\mathrm{Ba}^{2+}$ chloride solutions. J Colloid Interface Sci. 1984;97:446-55.

[33] Israelachvili JN, Adams GE. Measurement of forces between two mica surfaces in aqueous electrolyte solution in the range 0-100 nm. J Chem Soc, Faraday Trans 1. 1978;74:975-1001. [34] Rabinovich YI, Derjaguin BV, Churaev NV. Direct measurements of long-range surface forces in gas and liquid media. J Colloid Interface Sci. 1982;16:63-78.

[35] Ruckenstein E, Schiby D. On the origin of repulsive hydration forces between two mica plates. Chem Phys Letters. 1983;95.

[36] Abraham FF. The interfacial density profile of a Lennard-Jones fluid in contact with a (100) Lennard-Jones wall and its relationship to idealized fluid-wall systems: a Monte-Carlo simulation. J Chem Phys 1978;68 3713-6.

[37] Rao M, Berne BJ, Percus JK, Kalos MH. J Chem Phys. 1979;71:3802.

[38] Israelachvili JN, Pashley RM. Molecular layering of water at surfaces and origin of repulsive hydration forces. Nature. 1983;306:249-50.

[39] Ricci M, Spijker P, Voitchovsky K. Water-induced correlation between single ions imaged at the solid-liquid interface. Nat Commun. 2014;5.

[40] Leng Y. Hydration force between mica surfaces in aqueous $\mathrm{KCl}$ electrolyte solution. Langmuir. 2012;28:5339-49.

[41] Urbic T, Vlachy V, Dill KA. Confined water: A Mercedes-Benz model study. J Phys Chem B. 2006; 110:4963-70.

[42] Cherepanov DA. Force oscillations and dielectric overscreening of interfacial water. Phys Rev Lett. 2004;93.

[43] Henderson D, Lozadacassou M. A simple theory for the force between spheres immersed in a fluid. J Colloid Interface Sci. 1986;114:180-3.

[44] Li T-D, Gao J, Szoszkiewicz R, Landman U, Riedo E. Structured and viscous water in subnanometer gaps. Phys Rev B. 2007;75.

[45] Fenter P, Lee SS. Hydration layer structure at solid-water interfaces. MRS Bull. 2014;39:1056-61.

[46] Cheng L, Fenter P, Nagy KL, Schlegel ML, Sturchio NC. Molecular-scale density oscillations in water adjacent to a mica surface. Phys Rev Lett. 2001;87.

[47] Horn RG, Smith DT, Haller W. Surface forces and viscosity of water measured between silica sheets. Chem Phys Lett. 1989;162:404-8. 
[48] Vigil G, Xu ZH, Steinberg S, Israelachvili J. Interactions of silica surfaces. Journal of

502 Colloid and Interface Science. 1994;165:367-85.

503 [49] Drucker WA, Senden TJ, Pashley RM. Measurement of forces in liquids using a force microscope Langmuir. 1992;8:1831-6 [50] Shubin VE, Kekicheff P. Electrical double-layer structure revisited via a surface force apparatus - mica interfaces in lithium-nitrate solutions. J Colloid Interface Sci. 1993;155:108-23. [51] Ducker WA, Xu Z, Clarke DR, Israelachvili JN. Forces between alumina surfaces in saltsolutions - non-DLVO forces and the implications for colloidal processing. J Am Ceram Soc. 1994;77:437-43.

[52] Israelachvili JN. Intermolecular and surface forces. Third ed: Elsevier; 2011.

[53] Iler RK. The chemistry of silica. New York: Wiley; 1979.

[54] Claesson P, Horn RG, Pashley RM. Measurement of surface forces between mica sheets immersed in aqueous quaternary ammonium ion solutions. J Colloid Interface Sci. 1984;100:250-63.

[55] Ducker WA, Pashley RM. The forces between mica surfaces in ammonium-chloride solutions. J Colloid Interface Sci. 1989;131:433-9.

[56] Chapel JP. Electrolyte species dependent hydration forces between silica surfaces. Langmuir. 1994;10 4237-43.

[57] Drucker WA, Senden TJ, Pashley RM. Direct measurement of colloidal forces using atomic force microscope. Nature. 1991;353.

[58] Fielden ML, Hayes RA, Ralston J. Oscillatory and ion-correlation forces observed in direct force measurements between silica surfaces in concentrated $\mathrm{CaCl}_{2}$ solutions. Phys Chem Chem Phys. 2000;2:2623-8.

[59] Vakarelski IU, Ishimura K, Higashitani K. Adhesion between silica particle and mica surfaces in water and electrolyte solutions. Journal of Colloid and Interface Science. 2000;227:111-8. [60] Antognozzi M, Humphris ADL, Miles MJ. Observation of molecular layering in a confined water film and study of the layers viscoelastic properties. Appl Phys Lett. 2001;78:300-2. [61] Jeffery S, Hoffmann PM, Pethica JB, Ramanujan C, Ozer HO, Oral A. Direct measurement of molecular stiffness and damping in confined water layers. Phys Rev B. 2004;70.

[62] Acuna SM, Toledo PG. Short-range forces between glass surfaces in aqueous solutions. Langmuir. 2008;24:4881-7.

[63] Tulpar A, Van Tassel PR, Walz JY. Structuring of macroions confined between likecharged surfaces. Langmuir. 2006;22:2876-83.

[64] Acuna SM, Toledo PG. Nanoscale repulsive forces between mica and silica surfaces in aqueous solutions. J Colloid Interface Sci. 2011;361:397-9.

[65] Loh S-H, Jarvis SP. Visualization of ion distribution at the mica-electrolyte interface. Langmuir. 2010;26:9176-8.

[66] Kilpatrick JI, Loh S-H, Jarvis SP. Directly Probing the Effects of Ions on Hydration Forces at Interfaces. J Am Chem Soc. 2013;135:2628-34.

[67] Ducker WA, Senden TJ, Pashley RM. Measurement Of Forces In Liquids Using A Force Microscope. Langmuir. 1992;8:1831-6.

544

[68] Atkins DT, Ninham BW. Surface and structural forces measured between silica surfaces in 1,2-ethanediol. Colloids Surf, A. 1997;129:23-32. 
[69] Zeng Y, von Klitzing. R. Oscillatory forces of nanoparticle suspensions confined between rough surfaces modified with polyelectrolytes via the layer-by-layer technique. Langmuir. 2012;28:6313-21.

[70] Yang K, Lin Y, Lu X, Neimark AV. Solvation forces between molecularly rough surfaces. J Colloid Interface Sci. 2011;362:382-8.

[71] Akrami SMR, Nakayachi H, Watanabe-Nakayama T, Asakawa H, Fukuma T. Significant improvements in stability and reproducibility of atomic-scale atomic force microscopy in liquid. Nanotechnology. 2014;25. [72] Liang Y, Hilal N, Langston P, Starov V. Interaction forces between colloidal particles in liquid: Theory and experiment. Adv Colloid Interface Sci. 2007;134-35:151-66. [73] Valle-Delgado JJ, Molina-Bolivar JA, Galisteo-Gonzalez F, Galvez-Ruiz MJ, Feiler A, Rutland MW. Hydration forces between silica surfaces: Experimental data and predictions from different theories. Journal of Chemical Physics. 2005;123:12.

[74] Butt HJ. Measuring electrostatic, vanderwaals, and hydration forces in electrolyte-solutions with an atomic force microscope. Biophys J. 1991;60:1438-44.

[75] Meagher L. Direct measurement of forces between silica surfaces in aqueous $\mathrm{CaCl}_{2}$ solutions using an atomic force microscope. J Colloid Interface Sci. 1992;152:293-5. [76] Biggs S, Mulvaney P, Zukoski CF, Grieser F. Study of anion adsorption at the gold-aqueous solution interface by atomic-force microscopy. J Am Chem Soc. 1994;116:9150-7.

[77] Karaman ME, Pashley RM, Waite TD, Hatch SJ, Bustamante H. A comparison of the interaction forces between model alumina surfaces and their colloidal properties. Colloids and Surf, A. 1997;129:239-55. [78] Spaepen F. Structural model for the solid-liquid interface in monoatomic systems. Acta Mettallurgica. 1975;23:729-43.

[79] Curtin WA. Density-functional theory of the solid-liquid interface. Phys Rev Lett. 1987;59:1228-31.

[80] Richens DT. The Chemistry of Aqua-Ions: John Wiley \& Sons, Chichester; 1997. [81] Spohr E. Computer-simulation of the water platinum interface. J Phys Chem. 1989;93:617180.

[82] Spohr E. Computer-simulation of the water platinum interface - dynamic results. J Chem Phys. 1990;141:87-94.

[83] Delville A. Structure and properties of confined liquids - A molecular-model of the clay water interface. J Phys Chem. 1993;97:9703-12.

[84] Delville A, Sokolowski S. Adsorption of vapor at a solid interface - A molecular-model of clay wetting. J Phys Chem. 1993;97:6261-71.

[85] Lee SH, Rossky PJ. A comparison of the structure and dynamics of liquid water at hydrophobic and hydrophilic surfaces - a molecular-dynamics simulation study. J Chem Phys. 1994;100:3334-45.

[86] Vossen M, Forstmann F. The structure of water at a planar wall - an integral-equation approach with the central force model. Journal of Chemical Physics. 1994;101:2379-90. [87] Xia XF, Perera L, Essmann U, Berkowitz ML. The structure of water at platinum/water interfaces - molecular-dynamics computer-simulations. Surf Sci. 1995;335:401-15. [88] Bridgeman CH, Buckingham AD, Skipper NT, Payne MC. Ab-initio total energy study of uncharged 2:1 clays and their interaction with water. Mol Phys. 1996;89:879-88.

[89] Bridgeman CH, Skipper NT. A Monte Carlo study of water at an uncharged clay surface. J Phys Cond Mat. 1997;9:4081-7. 
[90] Akiyama R, Hirata F. Theoretical study for water structure at highly ordered surface: Effect of surface structure. J Chem Phys. 1998;108:4904-11.

[91] Yeh IC, Berkowitz ML. Structure and dynamics of water at water vertical bar Pt interface as seen by molecular dynamics computer simulation. J Electroanal Chem. 1998;450:313-25. [92] Stockelmann E, Hentschke R. A molecular-dynamics simulation study of water on $\mathrm{NaCl}(100)$ using a polarizable water model. J Chem Phys. 1999;110:12097-107. [93] Yeh IC, Berkowitz ML. Aqueous solution near charged Ag(111) surfaces: Comparison between a computer simulation and experiment. Chemical Physics Letters. 1999;301:81-6. [94] Wang J, Ocko BM, Davenport AJ, Isaacs HS. Insitu X-ray-diffraction and X-ray-reflectivity studies of the $\mathrm{Au}(111)$ electrolyte interface-reconstruction and anion adsorption. Phys Rev B. 1992;46 10321-38.

[95] Toney MF, Howard JN, Richer J, Borges GL, Gordon JG, Melroy OR, et al. Voltagedependent ordering of water-molecules at an electrode-electrolyte interface. Nature. 1994;368:444-6.

[96] Toney MF, Howard JN, Richer J, Borges GL, Gordon JG, Melroy OR, et al. Distribution of water-molecules at ag(111)/electrolyte interface as studied with surface X-ray-scattering. Surf Sci. 1995;335:326-32.

[97] Chu YS, Lister TE, Cullen WG, You H, Nagy Z. Commensurate water monolayer at the RuO2(110)/water interface. Phys Rev Lett. 2001;86:3364-7.

[98] Brown GE, Calas G, Waychunas GA, Petiau J. X-ray absorption-spectroscopy and its applications in mineralogy and geochemistry. Rev Mineral. 1988;18:431-512.

[99] Brown GE, Parks GA. Synchrotron-based x-ray absorption studies of cation environments in earth materials. Rev Geophys. 1989;27:519-33.

[100] Feidenhansl R. Surface-structure determination by x-ray-diffraction. Surf Sci Rep. 1989;10:105-88.

[101] Robinson IK, Tweet DJ. Surface X-ray-diffraction. Rep Prog Phys. 1992;55:599-651. [102] Brown GE, Parks GA. Sorption of trace elements on mineral surfaces: Modern perspectives from spectroscopic studies, and comments on sorption in the marine environment. Int Geol Rev. 2001;43:963-1073. [103] Bedzyk MJ, Cheng LW. X-ray standing wave studies of minerals and mineral surfaces: Principles and applications. In: Fenter PA, Rivers ML, Sturchio NC, Sutton SR, editors. Applications of Synchrotron Radiation in Low-Temperature Geochemistry and Environmental Sciences2002. p. 221-66.

[104] Huisman WJ, Peters JF, Zwanenburg MJ, deVries SA, Derry TE, Abernathy D, et al. Layering of a liquid metal in contact with a hard wall. Nature. 1997;390:379-81.

[105] Yu CJ, Richter AG, Kmetko J, Datta A, Dutta P. X-ray diffraction evidence of ordering in a normal liquid near the solid-liquid interface. Europhys Lett. 2000;50:487-93. [106] Yu CJ, Richter AG, Datta A, Durbin MK, Dutta P. Observation of molecular layering in thin liquid films using X-ray reflectivity. Phys Rev Lett. 1999;82:2326-9.

[107] Doerr AK, Tolan M, Schlomka JP, Press W. Evidence for density anomalies of liquids at the solid/liquid interface. Europhys Lett. 2000;52:330-6. [108] Yu CJ, Evmenenko G, Kmetko J, Dutta P. Effects of shear flow on interfacial ordering in liquids: X-ray scattering studies. Langmuir. 2003;19:9558-61. [109] Skipper NT, Soper AK, McConnell JDC. The structure of interlayer water in vermiculite. J Chem Phys. 1991;94:5751-60. 
[110] Karaborni S, Smit B, Heidug W, Urai J, vanOort E. The swelling of clays: Molecular simulations of the hydration of montmorillonite. Science. 1996;271:1102-4. [111] Sposito G, Skipper NT, Sutton R, Park SH, Soper AK, Greathouse JA. Surface geochemistry of the clay minerals. Proc Natl Acad Sci U S A. 1999;96:3358-64.

[112] Skipper NT, Williams GD, de Siqueira AVC, Lobban C, Soper AK. Time-of-flight neutron diffraction studies of clay-fluid interactions under basin conditions. Clay Miner. 2000;35:283-90. [113] Geissbuhler P, Fenter P, DiMasi E, Srajer G, Sorensen LB, Sturchio NC. Threedimensional structure of the calcite-water interface by surface X-ray scattering. Surf Sci. 2004;573:191-203.

[114] Fenter P, Sturchio NC. Calcite (104)-water interface structure, revisited. Geochim Cosmochim Acta. 2012;97:58-69.

[115] Zhou H, Ganesh P, Presser V, Wander MCF, Fenter P, Kent PRC, et al. Understanding controls on interfacial wetting at epitaxial graphene: Experiment and theory. Phys Rev B. 2012;85.

[116] Lee SS, Fenter P, Nagy KL, Sturchio NC. Monovalent ion adsorption at the muscovite (001)-solution interface: Relationships among ion coverage and speciation, interfacial water structure, and substrate relaxation. Langmuir. 2012;28:8637-50.

[117] Lee SS, Fenter P, Nagy KL, Sturchio NC. Changes in adsorption free energy and speciation during competitive adsorption between monovalent cations at the muscovite (001)water interface. Geochim Cosmochim Acta. 2013;123:416-26.

[118] Fenter P, Kerisit S, Raiteri P, Gale LD. Is the Calcite-Water Interface Understood? Direct Comparisons of Molecular Dynamics Simulations with Specular X-ray Reflectivity Data. J Phys Chem C. 2013;117:5028-42.

[119] Fenter P, Sturchio NC. Mineral-water interfacial structures revealed by synchrotron X-ray scattering. Prog Surf Sci. 2004;77:171-258.

[120] Binder H. The molecular architecture of lipid membranes - New insights from hydrationtuning infrared linear dichroism spectroscopy. Appl Spectrosc Rev. 2003;38:15-69. [121] Aman K, Lindahl E, Edholm O, Hakansson P, Westlund PO. Structure and dynamics of interfacial water in an L-alpha phase lipid bilayer from molecular dynamics simulations. Biophys J. 2003;84:102-15.

[122] Marsh D. Handbook of lipid bilayers. 2nd ed. Boca Raton, FL.: CRC Press, Taylor \& Francis Group; 2013. [123] Pandit SA, Bostick D, Berkowitz ML. Mixed bilayer containing dipalmitoylphosphatidylcholine and dipalmitoylphosphatidylserine: Lipid complexation, ion binding, and electrostatics. Biophys J. 2003;85:3120-31.

[124] Berkowitz ML, Bostick DL, Pandit S. Aqueous solutions next to phospholipid membrane surfaces: insights from simulations. Chemical reviews. 2006;106:1527-39. [125] Bockmann RA, Hac A, Heimburg T, Grubmuller H. Effect of sodium chloride on a lipid bilayer. Biophys J. 2003;85:1647-55.

[126] Pandit SA, Bostick D, Berkowitz ML. Mixed bilayer containing dipalmitoylphosphatidylcholine and dipalmitoylphosphatidylserine: lipid complexation, ion binding, and electrostatics. Biophys J. 2003;85:3120-31. [127] Schrader AM, Donaldson SH, Jr., Song J, Cheng CY, Lee DW, Han S, et al. Correlating steric hydration forces with water dynamics through surface force and diffusion NMR measurements in a lipid-DMSO-H2O system. Proceedings of the National Academy of Sciences of the United States of America. 2015;112:10708-13. 
[128] Yu J, Wei W, Danner E, Israelachvili JN, Waite JH. Effects of Interfacial Redox in Mussel Adhesive Protein Films on Mica. Adv Mater. 2011;23:2362-6.

[129] Rand RP, Fuller NL, Lis LJ. Myelin Swelling and Measurement of Forces between Myelin Membranes. Nature. 1979;279:258-60.

[130] Degennes PG. Penetration of a Coil into an Adsorbed Layer - Application to the Kinetics of Exchange and to Bridging Processes between Colloidal Particles. Cr Acad Sci Ii.

1985;301:1399-403.

[131] Degennes PG. Polymers at an Interface - a Simplified View. Adv Colloid Interfac. 1987;27:189-209.

[132] Harbich W, Servuss RM, Helfrich W. Passages in Lecithin-Water Systems. Z Naturforsch A. 1978;33:1013-7.

[133] Donaldson SH, Lee CT, Chmelka BF, Israelachvili JN. General hydrophobic interaction potential for surfactant/lipid bilayers from direct force measurements between light-modulated bilayers. Proceedings of the National Academy of Sciences of the United States of America. 2011;108:15699-704.

[134] Donaldson SH, Royne A, Kristiansen K, Rapp MV, Das S, Gebbie MA, et al. Developing a General Interaction Potential for Hydrophobic and Hydrophilic Interactions. Langmuir. 2015;31:2051-64.

[135] Sanchez-Iglesias A, Grzelczak M, Altantzis T, Goris B, Perez-Juste J, Bals S, et al. Hydrophobic Interactions Modulate Self-Assembly of Nanoparticles. Acs Nano. 2012;6:1105965.

[136] Aniansson EAG, Wall SN, Almgren M, Hoffmann H, Kielmann I, Ulbricht W, et al. Theory of Kinetics of Micellar Equilibria and Quantitative Interpretation of Chemical Relaxation Studies of Micellar Solutions of Ionic Surfactants. J Phys Chem-Us. 1976;80:905-22. [137] Aniansson GEA. Dynamics and Structure of Micelles and Other Amphiphile Structures. J Phys Chem-Us. 1978;82:2805-8.

[138] Banquy X, Kristiansen K, Lee DW, Israelachvili JN. Adhesion and hemifusion of cytoplasmic myelin lipid membranes are highly dependent on the lipid composition. BbaBiomembranes. 2012;1818:402-10.

[139] Donaldson S, Chmelka BF, Israelachvili JN. General hydrophobic interaction potential for surfactant/lipid bilayers from direct force measurements between light-modulated bilayers Proc Natl Acad Sci USA. 2011;108:15699-704.

[140] Mitchell DJ, Ninham BW. Curvature Elasticity of Charged Membranes. Langmuir. 1989;5:1121-3.

[141] Schrader AM, Donaldson SH, Song J, Cheng CY, Lee DW, Han S, et al. Correlating steric hydration forces with water dynamics through surface force and diffusion NMR measurements in a lipid-DMSO-H2O system. Proceedings of the National Academy of Sciences of the United States of America. 2015;112:10708-13.

[142] Schneck E, Sedlmeier F, Netz RR. Hydration repulsion between biomembranes results from an interplay of dehydration and depolarization. Proceedings of the National Academy of Sciences of the United States of America. 2012;109:14405-9.

[143] Israelachvili JN, Wennerstrom H. Entropic Forces between Amphiphilic Surfaces in Liquids. J Phys Chem-Us. 1992;96:520-31.

[144] Mcintosh TJ, Simon SA. Contributions of Hydration and Steric (Entropic) Pressures to the Interactions between Phosphatidylcholine Bilayers - Experiments with the Subgel Phase. Biochemistry-Us. 1993;32:8374-84. 
